\title{
Mental health support for children and adolescents with hearing loss: scoping review
}

Nathaniel Scherer, Tess Bright, David John Musendo, Timothy O'Fallon, Chris Kubwimana, Julian Eaton, Ritsuko Kakuma, Tracey Smythe and Sarah Polack

\section{Background}

Children with hearing loss are at increased risk of mental health conditions, including behavioural problems, but there is limited evidence about available mental health support.

\section{Aims}

We aimed to map the evidence on mental health support for children and adolescents with hearing loss.

\section{Method}

Medline, Embase, PsycINFO and grey literature databases were searched until April 2021. Articles of any study design were eligible if they described an intervention supporting the mental health of children with hearing loss. No restrictions were placed on geography or publication date. Four reviewers independently screened results by title, abstract and full text. Study characteristics and outcome data were extracted, with results narratively synthesised.

\section{Results}

From 5629 search results, 27 articles were included. A large majority of the studies $(81 \%, n=22)$ were from high-income settings, with two-thirds $(67 \%, n=18)$ conducted in the USA. Less than half $(41 \%, n=11)$ of the articles adopted experimental research designs, and the majority of studies included small samples. The interventions presented were diverse, with the majority either therapy based $(30 \%, n=8)$ or skills training $(30 \%$, $n=8$ ). Interventions included ice-skating, parent-child interaction therapy and resilience training. When measured, interventions demonstrated at least some evidence of effectiveness, although this was not always assessed with gold-standard methodology.

\section{Conclusions}

The evidence is lacking in breadth, study quality and geographical spread. That said, what is available indicates a range of effective approaches to support the mental health of children with hearing loss. Additional research is needed to improve the breadth of evidence on mental health support for this population.

\section{Keywords}

Childhood experience; outcome studies; psychosocial interventions; rehabilitation; primary care.

\section{Copyright and usage}

(c) The Author(s), 2021. Published by Cambridge University Press on behalf of the Royal College of Psychiatrists. This is an Open Access article, distributed under the terms of the creative Commons Attribution licence (https://creativecommons.org/ licenses/by/4.0/), which permits unrestricted re-use, distribution, and reproduction in any medium, provided the original work is properly cited.

\section{Background}

As of 2019 , an estimated $20 \%$ of the global population (or 1.57 billion) have hearing loss, with 430 million having moderate-tocomplete hearing loss. ${ }^{1}$ This is a $79 \%$ increase in reported rates from 1990, and by 2050, an estimated 2.45 billion people are estimated to have hearing loss, with 698 million having moderate-tocomplete hearing loss. Although hearing loss is more common among older adults, there are approximately 70 million children aged $0-15$ with hearing loss across the world.

Children with hearing loss may experience language deprivation, impacting development, communication and socioemotional skills. ${ }^{2,3}$ As a result, children with hearing loss are at increased risk of mental health conditions, such as anxiety and depression, with several studies demonstrating significantly higher prevalence of these conditions among children with hearing loss, compared with children without hearing loss. ${ }^{4-9}$ Evidence also shows that children with hearing loss are more likely to experience behavioural problems, including conduct and hyperactivity disorders. ${ }^{10,11}$ Half of mental health conditions start by the age of 14, yet these often go undetected and untreated. ${ }^{12}$ Among the general population, these conditions in childhood are associated with an increased risk of mental health concerns in adulthood, lower family income, lower probability of employment and lower probability of being married. ${ }^{13,14}$ Mental health conditions may also further disrupt ongoing child development, a challenge already present for children with hearing loss. Addressing mental health concerns, while promoting emotional, behavioural and psychological well-being, is imperative among children with hearing loss, in order to support a healthy childhood and reduce the risk of adverse experiences in adulthood.

Youth mental health has received growing attention in recent years, as evidenced by the inclusion of 104 network meta-analyses and meta-analyses of randomised controlled trials (RCTs) on mental health interventions for children and adolescents in a recent umbrella review published in $2021 .{ }^{15}$ However, there is limited synthesised evidence on interventions and types of support available for children with hearing loss that promote psychological, emotional and behavioural well-being, prevent mental health conditions and treat conditions that do arise. In 2019, a systematic review on the assessment and treatment of behavioural disorders in children with hearing loss found limited evidence of interventions to address behavioural problems. ${ }^{11}$ Evidence on interventions available for other mental health conditions is lacking. Information on the types of interventions applied, their characteristics and evidence of their effectiveness is needed to inform support programmes for children with hearing loss, whether these be in school, the community or delivered through the healthcare system.

\section{Aims}

This study aimed to systematically identify and map the evidence on mental health support for children and adolescents with hearing loss. We aimed to answer the following questions. 
(a) What are the characteristics of the available literature describing mental health support for children with hearing loss?

(b) What mental health interventions and support have been provided or evaluated for children with hearing loss?

(c) What evidence is available on the effectiveness of these interventions?

(d) What are the current gaps in the available evidence?

\section{Method}

With expected limited and heterogeneous data, we chose to conduct a scoping review, rather than an alternative evidence synthesis, in order to map the available evidence. ${ }^{16}$ The protocol for the review was registered on the Open Science Framework on 3 March 2020 (osf.io/8qdbz/). Ethics approval was obtained from the London School of Hygiene \& Tropical Medicine Research Ethics Committee (Ref: 19144).

The scoping review has been conducted and reported according to the PRISMA Extension for Scoping Reviews (PRISMA-ScR) guideline and followed Arksey \& O'Malley's framework for scoping reviews. ${ }^{17,18}$ We conducted the review across five stages: (a) identifying the research question; (b) identifying relevant studies; (c) study selection; (d) charting the data; and (e) collating and summarising the results.

\section{Eligibility criteria}

(a) Population: children aged 6-18, with diagnosed, proxy-report or self-reported hearing loss, of any severity. Included participants did not need to have a diagnosis of a mental disorder.

(b) Intervention: any initiative designed to improve the mental health and well-being of children with hearing loss, including interventions developed specifically for children with hearing loss or those adapted from interventions aimed at the general population. Interventions promoting developmental skills and protective factors, such as emotional regulation, resilience and self-esteem, were included if connection was made to child mental health and well-being. Interventions were also included if they addressed behavioural problems or disorders, such as hyperactivity or aggression. Activities could be focused on promotion, prevention or treatment, such as social interventions, skills development, targeted recreational activities and therapies. They could be delivered by any personnel in any setting. Interventions for parents, caregivers or other adults (for example teachers) were included if children with hearing loss were an intended beneficiary. Similarly, interventions were included if provided to a diverse group, as long as children with hearing loss were one of the beneficiaries.

(c) Comparator: studies with and without a control or comparison group were included. If a control or comparator is present, they must also be children with hearing loss of the same age, but who have not received the intervention.

(d) Outcomes: studies with or without assessment of any outcome were included. Where applicable, outcomes of interest included scores on mental health screening tools, acceptability/feasibility, cost-effectiveness and other reasonable data.

(e) Study design: published literature of any study design (quantitative, qualitative and mixed methods). Descriptive literature (i.e. without research methods applied) was included, if sufficient detail was provided on the intervention(s) available for children with hearing loss. Reviews and opinion pieces were excluded. There were no restrictions placed on geographic location, although articles needed to be in English. There were no limits placed on publication date. Grey literature, including dissertations and conference presentations, was included. Only articles with an available full text were included.

\section{Search strategy}

Articles were identified through a systematic search of Medline, Embase and PsycINFO. The search was initially conducted on 27 April 2020 and updated on 27 April 2021. An example of the search strategy is available in Supplementary File 1 available at https://doi.org/10.1192/bjo.2021.1045. Reference lists of each included study were examined in search of additional articles for inclusion.

The search for grey literature was conducted through OpenGrey and Google Scholar. Additionally, experts in this field were contacted for recommendations of known reports, and the websites of notable disability and hearing loss organisations were manually searched (Supplementary File 2).

\section{Study selection}

Authors N.S., T.B., D.J.M. and T.O.'F. independently screened all titles and abstracts against the eligibility criteria. Each record was screened by two reviewers. Eligible full-text articles were then independently reviewed by two reviewers. Although excluded from the final synthesis, systematic reviews and full-length books identified in the database search were manually screened by full-text, with included articles and book chapters selected for full-text screen, if relevant. Records identified from the reference lists of included articles were also filtered into the full-text screening. Discrepancies at any stage were discussed between the two reviewers, with a third and fourth reviewer consulted if needed. This review process was conducted using Covidence software.

\section{Data extraction and charting}

N.S. extracted the data for each study, using a custom form, developed in Excel. This extraction form was first piloted on three included articles, with amendments made as necessary. T.B., D.J. M. and T.O.'F. each independently reviewed one-third of data extracted. Discrepancies were discussed and resolved with N.S., with support from a third reviewer if needed. Data extracted included:

(a) Publication details: author, year of publication, title, country, aims/objectives, study design.

(b) Characteristics of mental health support: type (promotion, prevention, treatment), intended outcome, setting, delivery agent, intervention components.

(c) Outcomes: type (effectiveness, feasibility, acceptability, servicedelivery related), measurement tools, findings (both narrative and statistical).

\section{Quality assessment}

For an assessment of risk of bias, we used the critical appraisal tools from the Joanna Briggs Institute (JBI). There are various tools provided by JBI, designed for use with different designs, such as case report or RCT. Each tool can be used to determine the extent to which a study has addressed the possibility of bias in its design, conduct and analysis, via a series of relevant questions and standardised responses. ${ }^{19}$

Studies were assessed by N.S., with scores reviewed by S.P. Based on the assessment against a JBI checklist, each study was rated as having high, medium or low risk of bias.

(a) Low risk: all or almost all of the criteria were fulfilled, and those that were not fulfilled were thought unlikely to alter the conclusions. 


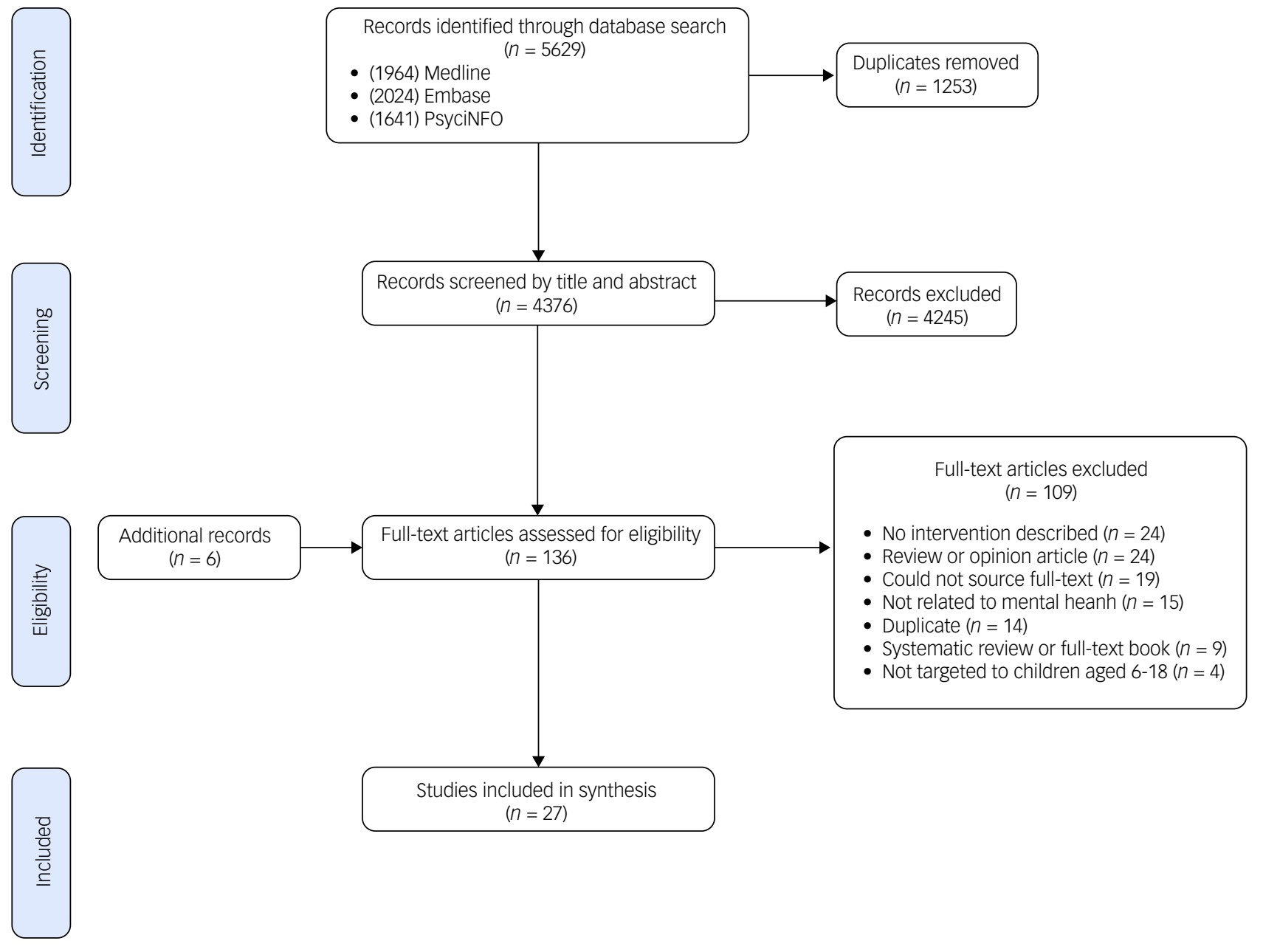

Fig. 1 PRISMA flow chart.

(b) Medium risk: some of the criteria were fulfilled, and those not fulfilled were thought unlikely to alter the conclusions of the study.

(c) High risk: few or no checklist criteria were fulfilled, and the conclusions of the study were thought likely to alter if these had been met.

\section{Synthesis of results}

Under the umbrella of mental health, the focus of this review, we have grouped articles by interventions that address psychological well-being and behavioural problems. Psychological well-being, in this instance, refers broadly to emotional well-being and mental health, and includes diagnosis or symptoms of mental health conditions, as well as related domains, such as resilience and self-esteem. Behavioural problems include disorders and concerns relating to disruptive and challenging behaviours, such as aggression, impulsivity and defiance. Specific behavioural disorders include attention-deficit hyperactivity disorder or oppositional defiant disorder.

Under these two subheadings, we grouped articles by intervention type. Findings have been summarised narratively, with the focus on the characteristics of the interventions. Information on effectiveness has been narratively presented, where this information was available.

When data presented in an article is disaggregated by age, with participants older or younger than the inclusion criteria included, only that attributable to children within the age range of 6-18 years was included in the synthesis.

\section{Results}

The database search generated 5629 results, from which 1253 duplicates were removed. In total, 4376 records were screened by title and abstract, from which 4245 were excluded based on the criteria above (Fig. 1). There were 130 full-text articles that were eligible for screening, with 6 additional records included from systematic reviews screened at full text $(n=3)$, records identified via the reference lists of included articles $(n=2)$ and the grey literature $(n=1)$. From 136 full texts assessed, 27 articles were included in the synthesis. $^{20-46}$

\section{Study characteristics}

Of the 27 included articles, 18 (67\%) come from North America, and specifically the USA (Table 1). Of the remaining, four (15\%) were conducted in Europe, four (15\%) the Middle-East, and one (4\%) in Asia. None were from Africa, Latin America or Australasia. Nine (33\%) were published after the year 2010, with $18(67 \%)$ prior to 2010 . Eleven (41\%) were published prior to the year 2000 .

Eighteen (67\%) provide information on support focused on psychological well-being, and related domains. Of these, four (15\%) targeted protective psychological factors (such as resilience and emotion management), one (4\%) social anxiety disorder, one (4\%) substance misuse disorder and one (4\%) obsessive-compulsive disorder. Fourteen (52\%) of the 27 included articles focused support 


\begin{tabular}{|c|c|c|}
\hline Variable and category & $n$ & $\%$ \\
\hline \multicolumn{3}{|l|}{ Region } \\
\hline Asia & 1 & 4 \\
\hline Europe & 4 & 15 \\
\hline Middle-East & 4 & 15 \\
\hline North America & 18 & 67 \\
\hline \multicolumn{3}{|l|}{ Country } \\
\hline Finland & 1 & 4 \\
\hline India & 1 & 4 \\
\hline Iran & 3 & 11 \\
\hline Poland & 1 & 4 \\
\hline Turkey & 1 & 4 \\
\hline UK & 2 & 7 \\
\hline USA & 18 & 67 \\
\hline \multicolumn{3}{|l|}{ Country income status } \\
\hline High & 22 & 81 \\
\hline Upper-middle & 4 & 15 \\
\hline Lower-middle & 1 & 4 \\
\hline \multicolumn{3}{|l|}{ Study design } \\
\hline Before and after & 3 & 11 \\
\hline Case report & 4 & 15 \\
\hline Case series & 5 & 19 \\
\hline Pilot study & 2 & 7 \\
\hline RCT & 6 & 22 \\
\hline Qualitative & 1 & 4 \\
\hline Descriptive & 6 & 22 \\
\hline \multicolumn{3}{|l|}{ Decade of publication } \\
\hline 1970 & 4 & 15 \\
\hline 1980 & 1 & 4 \\
\hline 1990 & 6 & 22 \\
\hline 2000 & 7 & 26 \\
\hline 2010 & 7 & 26 \\
\hline 2020 & 2 & 7 \\
\hline \multicolumn{3}{|l|}{ Source } \\
\hline Journal & 23 & 85 \\
\hline Book & 2 & 7 \\
\hline Thesis & 2 & 7 \\
\hline \multicolumn{3}{|l|}{ Mental health domain } \\
\hline Psychological well-being & 18 & 67 \\
\hline Behavioural problems & 14 & 52 \\
\hline \multicolumn{3}{|l|}{ Sample size } \\
\hline$\leq 10$ & 9 & 33 \\
\hline $11-20$ & 4 & 15 \\
\hline $21-50$ & 4 & 15 \\
\hline $51-100$ & 2 & 7 \\
\hline$>100$ & 2 & 7 \\
\hline N/A & 5 & 19 \\
\hline \multicolumn{3}{|l|}{ Risk of bias } \\
\hline Low & 7 & 26 \\
\hline Medium & 10 & 37 \\
\hline High & 4 & 15 \\
\hline N/A (description only) & 6 & 22 \\
\hline
\end{tabular}

on behavioural problems, such as conduct disorder. Some articles focused on both psychological well-being and behavioural problems and the percentages presented therefore total greater than $100 \%$.

Twenty (74\%) of the interventions included treatment options, 17 (63\%) promotion activities and 7 (26\%) prevention techniques. Eight (30\%) interventions were therapeutic in nature, nine (33\%) were a structured training programme, two (7\%) based on physical exercise, and one (4\%) medical. Seven (26\%) others were intervention packages, comprised of multiple single interventions.

Eighteen (67\%) described activities delivered in schools, nine (33\%) of which were special schools, three (11\%) special education classrooms in a mainstream school and one $(4 \%)$ a mainstream school. Four (15\%) were conducted in residential settings, with schooling on site. Six (22\%) were conducted in healthcare facilities, one (4\%) a community setting, and two (7\%) artificial/research settings. One (4\%) study did not provide information on the setting.

Nine $(33 \%)$ of the included articles presented a case report or case series, six (22\%) conducted randomised controlled trials, five (19\%) used quasi-experimental methods (of which two were pilot studies) and one (4\%) qualitative. Six (22\%) of the articles described an intervention or support structure, without any research methods applied. One-third (33\%) of articles included a sample size of less than ten participants, with only two studies $(7 \%)$ including more than 100 participants.

Summarising the ages and degree of hearing loss among participants included presented a challenge. Studies provided various classifications on the severity of hearing loss, and we have converted these under the same terms in Table 2. The ages of participants and severity of hearing loss overlapped across studies, and summary statistics were difficult to present with much coherence.

\section{Quality assessment}

The six (22\%) articles that describe (rather than evaluate) an intervention were not included in the quality assessment. Of the 21 remaining, $7(33 \%)$ were rated as having a low risk of bias, 10 (48\%) medium and 4 (19\%) high. Potential sources of bias among those rated to have a medium or high risk, included: inappropriate statistical analysis, including the absence of statistical power or sample size calculations in RCTs; no clinical information provided for participants in a case series; and unsuitable measurement of outcomes, including collection time points and screening method.

\section{Types of support}

\section{Psychological well-being}

As seen in Supplementary Table 1,17 articles targeted psychological well-being and related factors (for example resilience).

Five (29\%) of these focused on forms of counselling, psychotherapy or group support. ${ }^{28,31,37,43,44}$ Examples included peersupport groups and therapeutic play. Four (24\%) provided structured training and skills-based programmes, including group assertiveness training for social anxiety disorder. ${ }^{20,23,35,36}$ Two (12\%) were focused on physical exercise and related activities, including iceskating and dance lessons. ${ }^{26,30}$ One (6\%) study investigated the impact of hearing aids on psychological well-being. ${ }^{38}$

The remaining five $(29 \%)$ articles provided information on intervention 'packages' that included more than one intervention approach. ${ }^{22,24,40,45,46}$ These were typically delivered in healthcare or residential settings. Individual interventions described in the packages were typical of those provided in mental health programmes for the general population, and included cognitive-behavioural therapy, medication, art therapy and peer-support groups.

\section{Behavioural problems}

Fourteen articles provided support for behavioural problems. In four articles, the intervention or support provided addressed both psychological well-being and behavioural problems.

Five of the $14(36 \%)$ provided counselling or variations of psychotherapy, including video-counselling. ${ }^{25,28,39,42,44}$ Three (21\%) provided parents with training, and typically included skills-building exercises, using role-play and similar techniques. ${ }^{32,33,41}$ Three (21\%) focused training efforts on children. ${ }^{29,34,35}$ For example, the PATHS (Promoting Alternative Thinking Strategies) curriculum, which includes daily activities at school, delivered by teachers. ${ }^{34}$ One (7\%) supported physical activity, in this instance, iceskating. ${ }^{30}$ A further two (14\%) articles described a support package including various activities. ${ }^{21,27}$ This included Walden House, a residential programme for children with hearing loss 


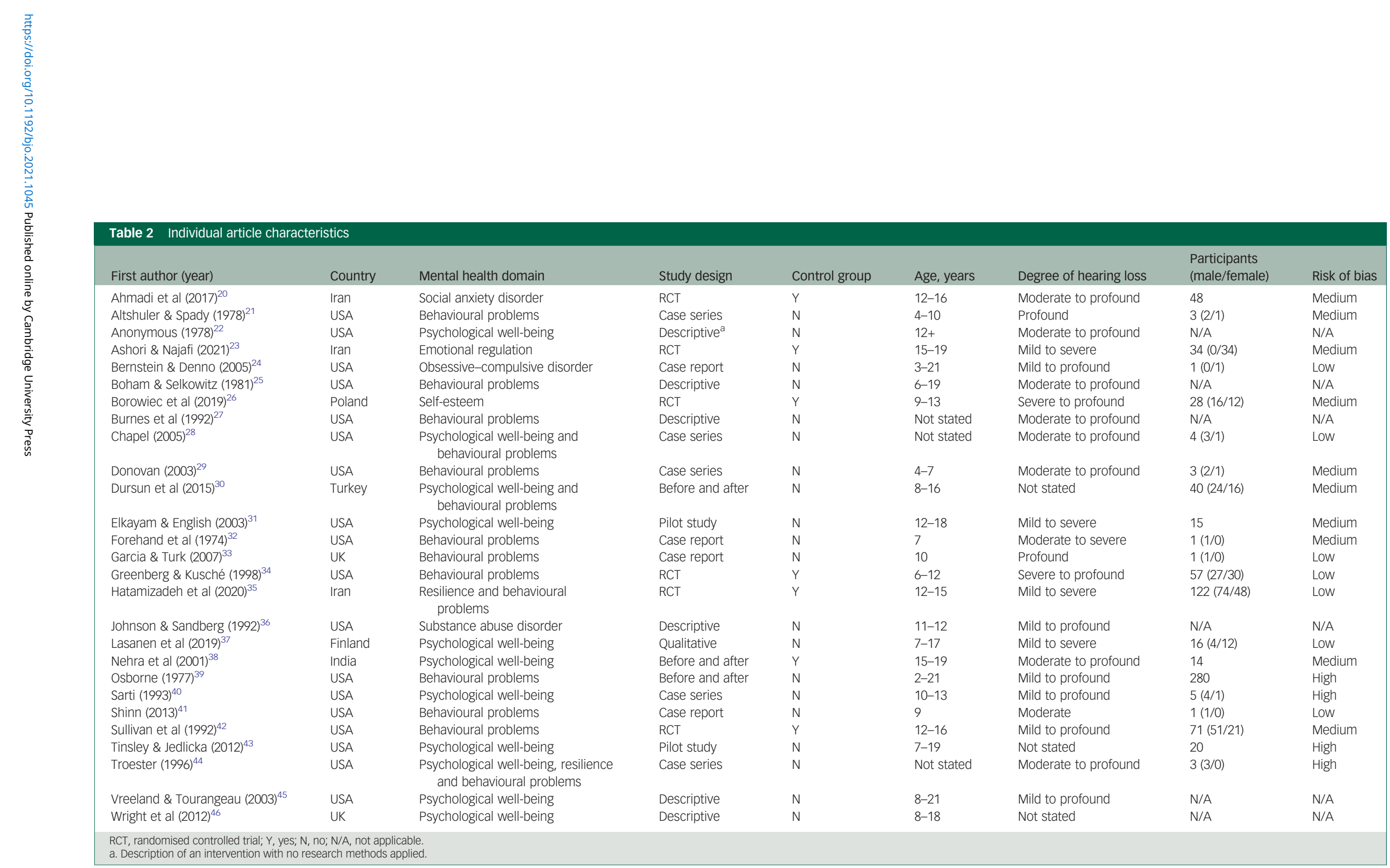


and behavioural problems, providing problem-solving skills, family therapy and role-play exercises. ${ }^{27}$

\section{Theoretical underpinnings}

Twenty three of the 27 articles provided information on the theory or evidence used to develop the intervention. The remaining four articles provided descriptions of logic-based arguments for the intervention model, without substantial information on evidence or theory. Of the 23 articles providing this information, 12 (52\%) provided evidence of previous use or effectiveness of the intervention among children without disabilities or hearing loss, four provided (17\%) evidence of its use with children with and without hearing loss, and four (17\%) articles provided evidence of the intervention among children with hearing loss only. Four (17\%) based the intervention on theories developed for children without hearing loss, such as the ABCD (Affective-Behaviour-CognitiveDynamic) model of child development.

\section{Adaptations made to interventions}

Of the 27 articles, 16 described specific considerations and adaptations made to the intervention to accommodate the needs of children with hearing loss, particularly when the support provided was initially developed for the general population.

Of these, 13 (81\%) described considerations of communication methods and needs, such as reading ability. Nine (56\%) used sign language or provided sign language interpretation. Three (19\%) made adaptations to the physical environment in which the intervention was delivered. For instance, seating groups in a circle to maximise visual contact, or providing pictures and posters to facilitate understanding. Three (19\%) adapted the core structure, components, tools and methods of established interventions. For example, established programmes for the general population, such as D.A.R.E. and the PATHS curriculum were adapted and prepared with support from hearing loss specialists.

\section{Outcomes}

Effectiveness

In total, 19 articles provided information on the effectiveness of the intervention or programme.

Eleven of these presented outcomes with regards to psychological well-being, with all but one finding some evidence of a positive impact of the intervention. Specifically, three (27\%) provided evidence on the effectiveness of training programmes for children with hearing loss, including resilience training. One of these, targeting social anxiety disorder, reported no significant differences in symptomology between intervention and control groups in children with profound hearing loss, but did see a difference for those with moderate hearing loss. Two (18\%) demonstrated the positive effect of physical activity interventions; dance lessons and iceskating. Therapy-based interventions were found beneficial by two $(18 \%)$ studies, one of which, a qualitative study, provided evidence of the positive perceived benefit from participants of regular peer-support groups, although changes in well-being were not measured nor observed with other standardised methods. One (9\%) other found no difference in participants pre- and post-intervention after receiving child-centred play therapy. One demonstrated significant improvements in well-being, including reduced symptoms of anxiety and depression, after children had used hearing aids for 6 months. Two studies (18\%) provided evidence on the effectiveness of intervention packages comprised of various approaches, including a package for obsessive-compulsive disorder. Of those described, five (45\%) demonstrated improvements in scores on quantitative standardised measurement tools (such as the Strengths and Difficulties Questionnaire) after the intervention was implemented, of which four (36\%) RCTs showed significant improvement in the intervention group and not the control group.

With regards to behavioural problems, 11 studies reported on outcomes, of which all but one found some evidence of a positive impact of the intervention. Five (45\%) found evidence on the benefit of training programmes, including parent behaviour training and teacher delivered resilience behaviour training for children. Three $(27 \%)$ demonstrated the positive impact of therapy-based interventions on behaviour, although one (9\%) showed no benefit. One study (9\%) showed improvements among children on the Strengths and Difficulties Questionnaire, including the behavioural domain, after regular ice-skating sessions. One (9\%) showed the benefit of a triadic intervention model for behavioural problems, focused on a core of reinforcement in schools. Seven (64\%) reported positive improvements in behavioural outcomes, assessed through standardised measurement tools. Three RCTs (27\%) demonstrated significant improvement in the intervention group and not the control, after delivery.

\section{Other outcomes}

Four articles provided evidence about the acceptability of the intervention to participants, delivery agents and/or parents. In one pilot study, this was the primary outcome that was measured quantitatively by questionnaire, with participants reporting good acceptance of the counselling method provided to support psychological wellbeing. In another, teachers reported feeling motivated to deliver the functional communication training for behavioural problems, and were happy with the training provided. In the third, both parents and delivery agents (clinicians) provided positive feedback in a directed workshop, with regards to the structure and components of the Webster-Stratton programme for behavioural problems. In the final case study of parent-child interaction therapy for behavioural problem, the mother expressed the value of learning to play with her child and manage their behaviour in a way that was fun and engaging for them.

\section{Discussion}

\section{Summary of evidence}

To the best of our knowledge, this is the first attempt to systematically identify and map the evidence on mental health support for children and adolescents with hearing loss. The scoping review identified 27 articles, two-thirds of which were conducted in the USA and two-thirds published before 2010. A number of interventions and support initiatives were described in the literature, including a broad range of therapy and counselling, and targeted training programmes, for both children and caregivers.

The interventions identified were, by and large, found to be effective at supporting the mental health and well-being of children with hearing loss. Although not all articles provided information on outcomes, nor all via quantitative assessment, those that did offered some encouraging signs as to the benefits of mental health support for children with hearing loss. Dance lessons, resilience training programmes and hearing aids demonstrate the diverse gamut of effective interventions that can be utilised to support children with hearing loss. Interventions provided treatment, prevention and promotion options in various settings, supporting recent advocacy for population- and community-level support for child mental health. ${ }^{47}$ As the evidence suggests, an effective and sustainable approach to child mental health includes support from an early age, improving information and awareness, providing social and emotional learning activities, increasing detection and identification, and improving access to treatment and rehabilitation. The 
interventions in this review provide some examples of interventions that may be effective within such a system, although as we will go on to discuss, the strength of the current evidence is limited.

Most of the interventions identified in this review were provided in school, which follows evidence from among the general population as to the benefits of integrated mental health provision within education systems. ${ }^{48}$ Interventions at school level provide a continuum of care that benefits a child's mental health, as well as their educational attainment. Only one identified intervention was conducted within a mainstream classroom, despite the recent global movement towards inclusive education. ${ }^{49}$ That said, a recent report outlines the limited transformations in education systems across the world, with most countries facing difficulties in inclusive provision, with understanding of what this means, and resources and evidence on good practice variable across regions. ${ }^{50}$ As reform continues to spread, there will need to be evidence gathered on appropriate mental health interventions in inclusive classrooms, to ensure children with hearing loss are not excluded.

When considering interventions for children with hearing loss, it is important to note components and delivery methods unique to this group. Many of the interventions were initially developed for use in the general population, and more than half of the included articles described interventions that had been developed from evidence for children without hearing loss and adapted for the needs of children with hearing loss. Adaptations were most commonly focused on communication, and often sign language. Communication is often cited as a major barrier and facilitator to accessing healthcare for people with hearing loss, and it is appropriate to see this as the focus of many adaptations. ${ }^{51}$ In some settings (typically highincome settings) such as the UK, and as seen in the included article from Wright et al (2012), specialist mental health services exist for children with hearing loss, where sign language and other accommodations are embedded in the service provided. ${ }^{46}$ However, in other settings, provision of communication technologies and sign language interpretation may not always be possible, given restricted resources. Often, and especially in low- and middle-income countries, mental health interventions are adapted from one context to another, in order to meet the culture, needs and resources of a population. In low- and middle-income countries, this often includes adapting interventions developed in high-income settings, although there are good examples of adaptation in the other direction, in which we see novel and effective mental health interventions developed in low-resource settings and adapted to those in high-income settings (an example being the Friendship Bench, developed in Zimbabwe and adapted to New York City in the USA). ${ }^{52}$ Adaptation is common and needed, and the well-evidenced stages of this process should be noted by those looking to adapt mental health interventions for children with hearing loss, in order to ensure appropriate intervention components and delivery mechanisms. This will include standard adaptations to the context, but also specific adaptation to the needs of children with hearing loss, such as those seen in this review. We did not find any studies that provided evidence of an adaptation process through formative research, theory of change workshop, feasibility study, or similar methods, and this would be a useful area of research going forwards, to promote interventions for children with hearing loss that are contextually and culturally appropriate, and hopefully then effective and sustainable. These approaches also promote the participation of stakeholders, including those with hearing loss and/or mental health conditions.

\section{Evidence gap}

Although 27 articles were identified, there is concern about the breadth, scope and strength of the evidence within the literature available. With two-thirds (67\%) of articles published before 2010, and $41 \%$ pre-2000, it is evident that the published literature on this topic is not growing at a fast rate. Interest and research in disability has steadily increased since the United Nations Convention on the Rights of Persons with Disabilities (2008), and calls for further evidence on scalable mental health interventions have been delivered by leading figures for many years. ${ }^{53}$ Despite an increased focus on global mental health and disability rights, this review suggests that mental health support for children with hearing loss is not gaining sufficient interest in the research community. The Lancet Commission on Global Mental Health and Sustainable Development, published in 2018, may stimulate additional research in the future, calling as it does for a focus on culturally appropriate and participatory approaches to translating evidence for promotion and care in mental health across diverse populations. ${ }^{54}$ Our approach to classification using a continuum from well-being to mental health diagnosis draws on these ideas. Exploring the evidence among adult populations is also needed given the higher prevalence of hearing loss in this age group.

Furthermore, nearly all (81\%) of the included articles come from high-income settings, with two-thirds (67\%) conducted in the USA. Just one (4\%) was conducted in a lower-middle or lowincome setting. There are no articles from Latin America or Africa, and just one study from Asia. The geographical scope of study into appropriate interventions for children with hearing loss must increase. Given that $80 \%$ of the world's population and people with disabilities are living in low- and middle-income countries, there is an urgent need for evidence on contextualised interventions. ${ }^{55}$ Relying on data from high-income settings has caused concern in the field of global mental health, as doing so reduces long-term effectiveness and sustainability of mental health interventions, especially when biomedical models of care are transferred to settings where they may not be culturally accepted, contextually feasible or appropriate. ${ }^{54}$ There is growing evidence on new and adapted mental health interventions for the general population in low- and middle-income settings, and as shown in this review, there is the possibility of adapting these to suit the needs of children with hearing loss (for example sign language provision). Evidence is needed on the process of adaptation and contextualisation, in order to inform delivery in the given country, and to provide a research framework for others.

Most of the studies were assessed to have medium or high risk of bias. Just six (22\%) of the studies available and included are RCTs. The majority of included articles offer interesting case reports, but these provide the rationale for larger-scale trials, rather than highquality evidence in and of themselves. Most of the studies included have also been conducted with very small sample sizes. Nearly half (48\%) of studies contained a sample size smaller than 20, increasing to two-thirds $(67 \%)$ when including those articles with no sample size available. To improve the evidence base with which to stimulate service provision and policy, there needs to be experimental research, with large samples, with which to build confident conclusions and inform scalable interventions. Potential sources of bias must be addressed, including appropriate statistical methods, including sample size calculations for sufficient power.

\section{Limitations}

There are several limitations to this review. First, we excluded articles that were not in the English language, and there may well be evidence missed that has been published in other languages. This is particularly important when considering the limited evidence from South America and East Asia. Our age range, 6-18 years, is broad and although not necessarily a limitation, it is important that readers of this review pay close attention to the targeted age range of interventions of interest. Younger children and older 
adolescents will respond differently to interventions, and will have different mental health needs, and not all interventions will be applicable across age groups. Second, we could not source 19 full-text articles, in which there may have been relevant and included studies. We contacted authors directly and utilised various institutional access agreements, but still could not find these for assessment. Third, we did not assess publication bias. When interpreting the results on effectiveness, readers should be conscious of the risk of positive publication bias and a potential lack of interest to publish results on ineffective interventions. Finally, in their framework for scoping reviews, Arksey \& O'Malley recommend an optional sixth step; consultation with stakeholders. ${ }^{18}$ Such consultation is time-consuming and costly, and was not feasible when undertaking this study, and may be an important area to address in any future and updated reviews.

\section{Implications}

This review has identified a number of articles and interventions, which generally showed positive results, but there are concerns over the breadth of the evidence available. There is evidence of some effective interventions, such as dance lessons and resilience training, but the applicability of the available evidence is limited by geographic location and publication date, and there is a need for more studies applying high-quality research methods. To improve the current evidence base, we must strengthen the quality of the research methods used and provide further research from low- and middle-income countries on adapting interventions to local contexts and on interventions tested at scale. Having a community of researchers set future research priorities, as informed by practitioners and people affected, will focus and strengthen research activities going forwards.

\section{Recommendations on mental health support for children with hearing loss}

Based on the findings of this review, we have listed below a number of considerations for delivering mental health support for children with hearing loss, including adaptations to existing mental health interventions.

(a) Consider the intervention models identified in this review, including the following.

(i) Peer-support: children with hearing loss can benefit from interaction with peers, reducing isolation, while helping them learn more about themselves and their experience as a child with hearing loss. Peer-support can act as both a treatment and health promotion option.

(ii) Resilience training: identified by a number of studies in this review, resilience training may be an effective way of preventing mental health conditions, especially if implemented at an early age. This type of training may be particularly appropriate to integrate into schools.

(iii) Emotional and behavioural management training: this type of structured training programme can be provided for both parents and children. Helping parents learn the tools with which to support their child's emotional regulation and behaviour may help create a home environment that promotes well-being and positive coping strategies. Training children themselves, possibly in school, will further help children with hearing loss build the toolkit with which to understand and approach the challenges they face.

(iv) Physical activity: physical activity is a simple way to build well-being and promote mental health. In this review, the physical interventions identified were specialist in nature - ice-skating and dance therapy are not necessarily available in all settings - but adapting these techniques and principles to a different context may prove effective.

(b) Consider the following when adapting or developing an intervention.

(i) Knowledge and awareness: it is important that the delivery agent is given appropriate training and sensitisation on disability and hearing loss. There may also be cause to provide information to children without hearing loss in the same setting, in order to reduce any apparent stigma or discrimination; for example, to hearing children in a mainstream classroom.

(ii) Communication methods: each child will have different communication needs, depending on their severity of hearing loss and personal preferences. Include appropriate training for delivery agents on communication methods, including clarity of speech and the language used. Where necessary, provide sign language interpretation, and where relevant provide training on hearing aid or cochlear implant use, as this knowledge will facilitate improved communication and reduce stigma.

(iii) Physical environment: consider the environment in which the intervention is delivered. This may be a mainstream classroom, for instance, that is not set up to accommodate the needs of children with hearing loss. Considering the configuration of seating in a group is one example; children sitting in a circle may help improve visual contact and support those who lipread. Keeping the environment quiet and free from distractions will also help children with hearing loss, as might keeping the door closed and adding soft material to the underside of chairs.

(iv) Intervention materials and techniques: where suitable, use visual aids and cues to support the intervention components. One example may include pictures matched to emotions, to aid the understanding and communication of feelings. Other techniques to reinforce behaviours and actions may include role-play, storytelling and peerfeedback.

(c) Consider the following during intervention development and implementation.

(i) Interventions identified in this review benefitted from clear communication and coordination between different groups involved. For instance, interventions in schools were strengthened when teachers and parents worked together.

(ii) Conduct feasibility studies and pilot studies, where possible, in order to make any necessary amendments early in the implementation process. This will also help inform others in the future.

(iii) Providing a range of interventions within a service or facility (such as a school), where this is feasible, may provide the best model with which to support a personalised approach for each child.

(d) Consultation and coordination may help promote feasible, acceptable and sustainable intervention programmes.

(i) Consult the children, their parents, mental health and hearing specialists, and the delivery agents (such as teachers). Understand their needs and context in the adaptation or development phase.

(ii) In all, talk to the children who are the intended target of the intervention about their own needs and preferences for intervention components, including communication methods. 
Nathaniel Scherer (D), International Centre for Evidence in Disability, London School of Hygiene \& Tropical Medicine, UK; Tess Bright, International Centre for Evidence in Disability, London School of Hygiene \& Tropical Medicine, UK; David John Musendo International Centre for Evidence in Disability, London School of Hygiene \& Tropical Medicine, UK; Timothy O'Fallon, International Centre for Evidence in Disability, London school of Hygiene \& Tropical Medicine, UK; Chris Kubwimana, DeafReach, UK; Julian Eaton (D. Centre for Global Mental Health, London School of Hygiene \& Tropical

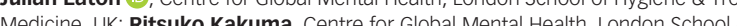
Hygiene \& Tropical Medicine, UK; Tracey Smythe, International Centre for Evidence in Hygiene \& Tropical Medicine, UK; Tracey Smythe, International Centre for Evide
Disability, London School of Hygiene \& Tropical Medicine, UK; Sarah Polack International Centre for Evidence in Disability, London School of Hygiene \& Tropical Medicine, UK

Correspondence: Nathaniel Scherer. Email: nathaniel.scherer@|shtm.ac.uk

First received 20 Jul 2021, final revision 20 Sep 2021, accepted 11 Oct 2021

\section{Supplementary material}

Supplementary material is available online at https://doi.org/10.1192/bjo.2021.1045

\section{Data availability}

Data availability is not applicable to this article as no new data were created or analysed in this study

\section{Acknowledgements}

The search string for this review was compiled with support from an Information Specialist at the London school of Hygiene \& Tropical Medicine.

\section{Author contributions}

N.S., T.B., C.K., J.E., R.K., T.S. and S.P. conceptualised the research question, design and meth odology. N.S., T.B., D.J.M. and T.O.'F. acquired, analysed and interpreted the data, with support from all authors. S.P. provided review of the quality appraisal. R.K., T.S. and S.P. provided supervision throughout. N.S. drafted the manuscript, with input and critical review from all authors. All authors read and approved the final manuscript.

\section{Funding}

This work was supported by CBM International and BMZ (Bundesministerium für wirtschaftliche Zusammenarbeit und Entwicklung) under the project 'Improving the psychosocial resilience of children with and without disabilities in the Gaza Strip'. Funding for the Covidence systematic review software was provided through the PENDA project (Programme for Evidence to Inform Disability Action) funded by the UK's Foreign Commonwealth and Development Office.

\section{Declaration of interest}

\section{References}

1 Haile LM, Kamenov K, Briant PS, Orji AU, Steinmetz JD, Abdoli A, et al. Hearing loss prevalence and years lived with disability, 1990-2019: findings from the Global Burden of Disease Study 2019. Lancet 2021; 397: 996-1009.

2 Briscoe J, Bishop DV, Norbury CF. Phonological processing, language, and literacy: a comparison of children with mild-to-moderate sensorineural hearing loss and those with specific language impairment. J Child Psychol Psychiatry 2001; 42: 329-40.

3 Calderon R, Greenberg M. Social and Emotional Development of Deaf Children: Family, School, and Program Effects. Oxford University Press, 2011.

4 Ariapooran S. Relationship between mother-child interaction and symptoms of anxiety disorders in deaf and hard-of-hearing children. Empowering Except Child 2018; 9: 51-63.

5 Gentili N, Holwell A. Mental health in children with severe hearing impairment. Adv Psychiatr Treat 2018; 17: 54-62.

6 Lawrence BJ, Jayakody DMP, Bennett RJ, Eikelboom RH, Gasson N, Friedland PL. Hearing loss and depression in older adults: a systematic review and meta-analysis. Gerontologist 2020; 60: e137-54.

7 Shoham N, Lewis G, Favarato G, Cooper C. Prevalence of anxiety disorders and symptoms in people with hearing impairment: a systematic review. Soc Psychiatry Psychiatr Epidemiol 2019; 54: 649-60.

8 Theunissen SC, Rieffe C, Kouwenberg M, De Raeve L, Soede W, Briaire JJ, et al. Anxiety in children with hearing aids or cochlear implants compared to normally hearing controls. Laryngoscope 2012; 122: 654-9.

9 Theunissen SC, Rieffe C, Kouwenberg M, Soede W, Briaire JJ, Frijns JH. Depression in hearing-impaired children. Int J Pediatr Otorhinolaryngol 2011; 75: 1313-7.
10 Stevenson J, Kreppner J, Pimperton H, Worsfold S, Kennedy C. Emotional and behavioural difficulties in children and adolescents with hearing impairment: a systematic review and meta-analysis. Eur Child Adolesc Psychiatry 2015; 24: 477-96.

11 Bigler D, Burke K, Laureano N, Alfonso K, Jacobs J, Bush ML. Assessment and treatment of behavioral disorders in children with hearing loss: a systematic review. Otolaryngol Head Neck Surg 2019; 160: 36-48.

12 Kessler RC, Berglund $P$, Demler $O$, Jin $R$, Merikangas $K R$, Walters $E E$. Lifetime prevalence and age-of-onset distributions of DSM-IV disorders in the National Comorbidity Survey Replication. Arch Gen Psychiatry 2005; 62: 593-602.

13 Clark C, Rodgers B, Caldwell T, Power C, Stansfeld S. Childhood and adulthood psychological ill health as predictors of midlife affective and anxiety disorders: the 1958 British Birth Cohort. Arch Gen Psychiatry 2007; 64: 668-78.

14 Goodman A, Joyce R, Smith JP. The long shadow cast by childhood physical and mental problems on adult life. Proc Natl Acad Sci 2011; 108: 6032.

15 Correll CU, Cortese S, Croatto G, Monaco F, Krinitski D, Arrondo G, et al. Efficacy and acceptability of pharmacological, psychosocial, and brain stimulation interventions in children and adolescents with mental disorders: an umbrella review. World Psychiatry 2021; 20: 244-75.

16 Munn Z, Peters MDJ, Stern C, Tufanaru C, McArthur A, Aromataris E. Systematic review or scoping review? Guidance for authors when choosing between a systematic or scoping review approach. BMC Med Res Methodol 2018; 18: 143.

17 Tricco AC, Lillie E, Zarin W, O'Brien KK, Colquhoun $\mathrm{H}$, Levac D, et al. PRISMA extension for scoping reviews (PRISMA-SCR): checklist and explanation. Ann Intern Med 2018; 169: 467-73.

18 Arksey H, O'Malley L. Scoping studies: towards a methodological framework. Int J Soc Res Methodol 2005; 8: 19-32.

19 Moola S, Munn Z, Tufanaru C, Aromataris E, Sears K, Sfetcu R, et al. Chapter 7 : Systematic reviews of etiology and risk. In JBI Manual for Evidence Synthesis (eds E Aromataris and Z Munn). JBI, 2020.

20 Ahmadi H, Daramadi PS, Asadi-Samani M, Givtaj H, Sani MRM. Effectiveness of group training of assertiveness on social anxiety among deaf and hard of hearing adolescents. Int Tinnitus J 2017; 21: 14-20.

21 Altshuler KZ, Spady F. The emotionally disturbed deaf child: a first program of research and therapy. J Commun Disord 1978; 11: 171-86.

22 Anonymous. Gold award: mental health services for the deaf-St. Elizabeths Hospital, Washington, D.C. Hosp Community Psychiatry 1978; 29: 674-7.

23 Ashori M, Najafi F. Emotion management: life orientation and emotion regulation in female deaf students. J Child Fam Stud 2021; 30: 723-32.

24 Bernstein V, Denno LS. Repetitive behaviors in CHARGE syndrome: differential diagnosis and treatment options. Am J Med Genet2005; 133: 232-9.

25 Boham BE, Selkowitz S. Video-counseling for multi-handicapped hearingimpaired students. Am Ann Deaf 1981; 126: 587-90.

26 Borowiec J, Hökelmann A, Osiński W. The level of self-esteem of deaf children: can participating in dance lessons with vibrational headphones improve it? Arts Psychother 2019; 64: 34-8.

27 Burnes S, Seabolt D, Vreeland J. Deaf culturally affirmative programming for children with emotional and behavioral problems. JADARA 2019; 26 : 12-17.

28 Chapel SL. Child-Centered Play Therapy with Deaf Children: Exploring Linguistic and Cultural Implications (Counselor Education Master's Theses). The College at Brockport: State University of New York, 2005.

29 Donovan AL. Functional Communication Training in Young Deaf Children: Effects of Reinforcement Magnitude on Requesting and Time-on Task (PhD Doctoral Thesis). Faculty of the Graduate School, University of Minnesota, 2003.

30 Dursun OB, Erhan SE, Ibiş E, Esin IS, Keleş S, Şirinkan A, et al. The effect of ice skating on psychological well-being and sleep quality of children with visual or hearing impairment. Disabil Rehabil 2015; 37: 783-9.

31 Elkayam J, English K. Counseling adolescents with hearing loss with the use of self-assessment/significant other questionnaires. J Am Acad Audiol 2003; 14 $485-99$.

32 Forehand R, Cheney $\mathrm{T}$, Yoder P. Parent behavior training: effects on the noncompliance of a deaf child. J Behav Ther Exp Psychiatry 1974; 5: 281-3.

33 Garcia R, Turk J. The applicability of Webster-Stratton Parenting Programmes to deaf children with emotional and behavioural problems, and autism, and their families: annotation and case report of a child with autistic spectrum disorder. Clin Child Psychol Psychiatry 2007; 12: 125-36.

34 Greenberg MT, Kusché CA. Preventive intervention for school-age deaf children: the PATHS curriculum. J Deaf Stud Deaf Educ 1998; 3: 49-63.

35 Hatamizadeh N, Adibsereshki N, Kazemnejad A, Sajedi F. Randomized trial of a resilience intervention on resilience, behavioral strengths and difficulties of mainstreamed adolescent students with hearing loss. Int J Pediatr Otorhinolaryngol 2020; 128: 109722. 
36 Johnson R, Sandberg K. Drug Abuse Resistance Education (D.A.R.E): special consideration for students who are deaf or hard of hearing. JADARA 1992; 26: 9-11

37 Lasanen M, Määttä K, Uusiautti S. 'I am not alone' - an ethnographic research on the peer support among northern-Finnish children with hearing loss. Early Child Dev Care 2019; 189: 1203-18.

38 Nehra A, Mann SB, Sharma SC, Verma SK. Effect of hearing AIDS on psycho social dysfunctioning. Indian J Otolaryngol Head Neck Surg 2001; 53: 291-4.

39 Osborne JG. Installing and maintaining a behavior modification system in a school for the deaf. Rehabil Psychol 1977; 24: 1-58.

40 Sarti DM. Reaching the deaf child: a model for diversified intervention. Smith Coll Stud Soc Work 1993; 63: 187-98.

41 Shinn MM. Parent-child interaction therapy with a deaf and hard of hearing family. Clin Case Stud 2013; 12: 411-27.

42 Sullivan PM, Scanlan JM, Brookhouser PE, Schulte LE, Knutson JF. The effects of psychotherapy on behavior problems of sexually abused deaf children. Child Abuse Negl 1992; 16: 297-307.

43 Tinsley $\mathrm{K}$, Jedlicka H. Equine Assisted Counseling With Deaf Families. In Harnessing the Power of Equine Assisted Counseling: Adding Animal Assisted Therapy to Your Practice (ed. K Trotter). Routledge, 2012.

44 Troester JD. A therapeutic play group for children with hearing impairments. J Child Adolesc Group Ther 1996; 6: 101-9.

45 Vreeland J, Tourangeau J. Culturally affirmative residential treatment services for deaf children with emotional and behavioral disorders. In Mental Health Care of Deaf People: A Culturally Affirmative Approach (eds Glickman NS, Gulati S): 239-60. Lawrence Erlbaum Associates Publishers, 2003.

46 Wright B, Walker R, Holwell A, Gentili N, Barker M, Rhys-Jones S, et al. A new dedicated mental health service for deaf children and adolescents. Adv Ment Health 2012; 11: 95-105.
47 Petersen I, Evans-Lacko S, Semrau M, Barry MM, Chisholm D, Gronholm P, et al. Promotion, prevention and protection: interventions at the population- and community-levels for mental, neurological and substance use disorders in low- and middle-income countries. Int J Ment Health Syst 2016; 10: 30.

48 Fazel M, Hoagwood K, Stephan S, Ford T. Mental health interventions in schools 1: mental health interventions in schools in high-income countries. Lancet Psychiatry 2014; 1: 377-87.

49 Schuelka M, Johnstone CJ. Global trends in meeting the educational rights of children with disabilities: from international institutions to local responses. Reconsidering Dev 2012; 2: 1-14.

50 United Nations Educational Scientific and Cultural Organization. Towards Inclusion in Education: Status, Trends and Challenges. UNESCO, 2020.

51 Kuenburg A, Fellinger P, Fellinger J. Health care access among deaf people. J Deaf Stud Deaf Educ 2015; 21: 1-10.

52 Rosenberg T. Depressed? Here's a Bench. Talk to Me. New York Times. 22 Jul 2019.

53 Patel V, Sartorius N. From science to action: the Lancet Series on Global Mental Health. Curr Opin Psychiatry 2008; 21: 109-13.

54 Patel V, Saxena S, Lund C, Thornicroft G, Baingana F, Bolton P, et al. The Lancet Commission on global mental health and sustainable development. Lancet 2018; 392: 1553-98.

55 World Bank, World Health Organization. The World Report on Disability. WHO, 2011. 